\title{
A case report of successful treatment of pyoderma gangrenosum in a patient with autoimmune hepatitis, and review of the literature
}

\author{
Theodoros Androutsakos ${ }^{1 *}$, Paraskevas Stamopoulos ${ }^{2}$, Kiriaki Aroni $^{3}$ and Gregorios Hatzis ${ }^{1}$
}

\begin{abstract}
Background: Pyoderma Gangrenosum (PG) is a cutaneous condition, its diagnosis suggested by the presence of a painful cutaneous ulcer showing rapid progression. Pyoderma gangrenosum is associated with a concomitant systemic disease in 50 to $70 \%$ of cases, including inflammatory bowel disease (IBD), rheumatoid arthritis, and lymphoproliferative disorders. Although PG has also been reported with viral hepatitis, it is rarely associated with autoimmune hepatitis.

Case presentation: A 19-year-old Caucasian female, with a prior diagnosis of autoimmune hepatitis (AlH) in remission, presented with bilateral lower limb ulcers 4 years after the diagnosis of AlH. She was diagnosed with PG and treated with high-dose prednisolone, methotrexate and cyclosporine. One year later she was well, the ulcers completely healed, and with the autoimmune hepatitis still in remission.

Conclusion: We report a case of autoimmune hepatitis and the subsequent, rarely occurring, extra-hepatic onset of pyoderma gangrenosum, with the AlH in remission, strengthening the association between the two conditions. Since both the AlH and the PG can present serious diagnostic challenges, thus delaying vital therapy, it is important that the development of either prompts us to consider the possibility of the other developing in the future or if already present facilitate its diagnosis, such considerations making the case for a systematic follow up.
\end{abstract}

Keywords: Autoimmune hepatitis, Pyoderma gangrenosum, Methotrexate, Cyclosporine

\section{Background}

Pyoderma Gangrenosum (PG), classified as a neutrophilic dermatosis, is an ulcerative cutaneous condition, first described in 1930 by Brusting and colleagues [1, 2]. Commonly, the lesions present as tender pustules that evolve into enlarging suppurative ulcers, with a tendency to last for months or years.

Although of non-infectious etiology, the pathogenesis of the disease is unclear, but evidence suggests an underlying defective neutrophilic function [1, 3]. After excluding more common causes of cutaneous ulcerations, the diagnosis of PG is suggested by the presence of a painful, necrolytic cutaneous ulcer, with an irregular undermined border, showing rapid progression [1]. Histologically, there is lymphocytic infiltration in the early stages followed by neutrophilic infiltration and hemorrhage $[4,5]$. Pyoderma

\footnotetext{
*Correspondence: T_Androutsakos@yahoo.gr

'Pathophysiology Department, Laiko General Hospital, National and

Kapodistrian University of Athens, Medical School, Athens, Greece

Full list of author information is available at the end of the article
}

gangrenosum is associated with a concomitant disease in 50 to $70 \%$ of cases, including inflammatory bowel disease (IBD) in 10 to $15 \%$ [6], rheumatoid arthritis [7], and lymphoproliferative disorders [8-10]. Although PG has been encountered with viral hepatitis [11], only a small number of cases have been reported with autoimmune hepatitis (AIH) [12-15]-one associated with concurrent primary sclerosing cholangitis and ulcerative colitis [13].

Treatment of PG is non-surgical and consists of a combination of local wound care and systemic therapy, the latter centered on the use of high-dose corticosteroids [16]. Other treatment agents include immunosuppressants, intravenous immunoglobulin, and biologic agents-such as tumor necrosis factor alpha inhibitors [16]. We report a 19-year-old patient, with a history of $\mathrm{AIH}$ in remission, who presented with pyoderma gangrenosum of the lower extremities, and was treated successfully with cortisone, cyclosporine and methotrexate. 


\section{Case presentation Clinical aspects}

A 19-year-old Caucasian female presented to us with bilateral lower limb ulcers. She reported a scratch on her left ankle 3 months earlier that worsened over time into an ulcer, in spite of antibiotic treatment. A week later, new lesions appeared on the left leg and similar ones were seen on her right leg. The patient denied fever, weight loss, or other signs or symptoms of systemic illness. Four years earlier she had been admitted at another hospital with transaminasemia and jaundice and was diagnosed with autoimmune hepatitis based on serological (ANA and ASMA) positivity and compatible histopathological features, in the absence of viral markers. Treatment with prednisolone and azathioprine brought the disease into remission. The patient was on $5 \mathrm{mg}$ prednisolone upon presentation.

On admission, the patient was afebrile, bearing purulent, painful ulcers in both legs (Fig. 1a). Lab results showed the inflammation markers just exceeding the upper normal limits (Table 1). Transaminases were within a normal range, while anti-nuclear antibodies (ANA) were positive at a titer of $1 / 320$ and anti-smooth muscle antibodies (ASMA) were positive at a low titer (1/80). Cryoglobulin test was negative. The lesions were cultured for bacteria and Mycobacterium tuberculosis, and empiric therapy was started with clindamycin and moxifloxacin. Magnetic Resonance Imaging (MRI) of the region of ulcers in both legs revealed diffuse subcutaneous oedema and subcutaneous nodular lesions on the dorsal surface of the feet. With a working diagnosis of pyoderma gangrenosum, a skin biopsy was acquired, her previous medication of azathioprine was reintroduced at $150 \mathrm{mg}$, while the steroids were increased from 5 to $20 \mathrm{mg}$ prednisolone, daily. The patient was discharged pending the pathology and bacteriology results.

She returned a week later with arthritis of both ankles and worsening of the ulcers (Fig. 1b). Further cultures were again negative. The biopsy was interpreted as being compatible with pyoderma gangrenosum (Fig. 2a, b), hence prednisolone $1 \mathrm{mg} / \mathrm{kg}$ was started, azathioprine was switched to cyclosporine at $150 \mathrm{mg}$ that was further increased to $250 \mathrm{mg}$ daily, and methotrexate $10 \mathrm{mg} /$ week was added to the regimen. Given the rare combination of pyoderma gangrenosum with autoimmune hepatitis, upper and lower endoscopies, as well as upper abdominal MRI and Magnetic Resonance Cholangio-pancreatography (MRCP) were done to exclude more common associated diseases. All of the investigations were negative. A review of the initial liver biopsies confirmed the diagnosis of autoimmune hepatitis, showing dense portal and periportal lymphocytic and plasmacytic infiltrates, as well as mild periportal fibrosis. The patient was again discharged, with slow tapering of the steroids.

On follow up a month later she was feeling well, with no ankle swelling, and with all ulcers healing satisfactorily (Fig. 1c). Six months hence she was on $4 \mathrm{mg}$ methylprednisolone, $100 \mathrm{mg}$ cyclosporine, and $7.5 \mathrm{mg}$ methotrexate, with normal liver enzymes, and with the ulcers nearly healed (Fig. 1d). Cyclosporine was discontinued at 8 months, and a year later the patients was on $4 \mathrm{mg}$ methylprednisolone and $7.5 \mathrm{mg}$ methotrexate, with further improvement and complete healing of the ulcers (Fig. 1e).

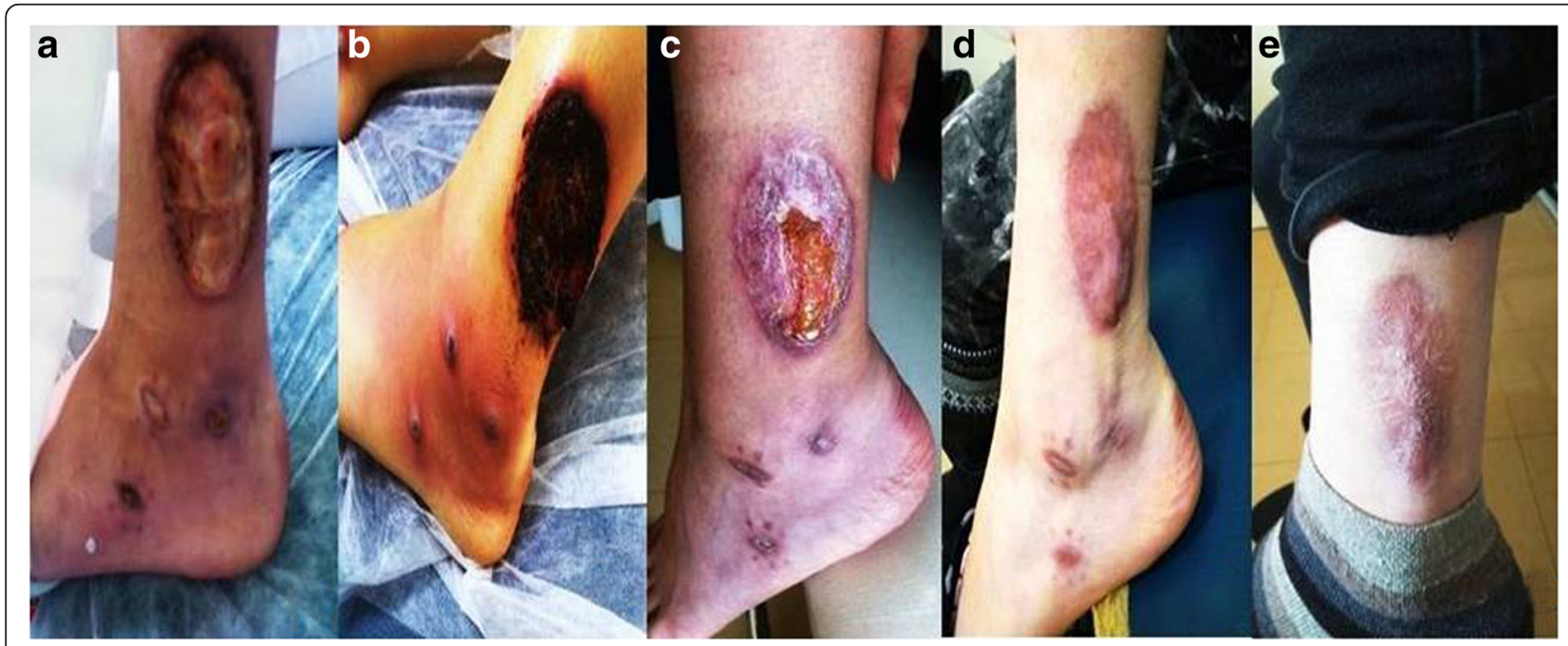

Fig. 1 Images of ulcerated areas. a On admission. b A week later. The swelling and the inflammation are noted. c One month later. Significant improvement of the ulcerating areas. $\mathbf{d}$ At 6 months. The inflammation has subsided, with further improvement of the ulcerating areas. e At one year. No new ulcers and healed old ones 
Table 1 Patient's lab results

\begin{tabular}{|c|c|c|}
\hline Lab test & Normal values & Result \\
\hline $\mathrm{Hgb}(\mathrm{g} / \mathrm{dl})$ & $12,0-16,0$ & 12,4 \\
\hline Hct (\%) & $38-47$ & 37,7 \\
\hline $\mathrm{WBC}(\mathrm{K} / \mu \mathrm{L})$ & $4,5-11,0$ & 12,300 \\
\hline Neut/Lymph/Mono/Eos (\%) & & $69 / 25 / 5 / 0$ \\
\hline $\mathrm{PLT}(\mathrm{K} / \mu \mathrm{L})$ & $140-440$ & 357 \\
\hline Glu (mg/dl) & 74-106 & 75 \\
\hline Urea (mg/dl) & $15-40$ & 25 \\
\hline Creatinine (mg/dl) & $0,6-1,1$ & 0,7 \\
\hline AST (U/L) & $5-31$ & 13 \\
\hline $\mathrm{ALT}(\mathrm{U} / \mathrm{L})$ & $5-34$ & 11 \\
\hline$\gamma G T(U / L)$ & $7-36$ & 12 \\
\hline $\operatorname{ALP}(U / L)$ & $48-141$ & 59 \\
\hline INR & $0,9-1,15$ & 1 \\
\hline HBA1C (\%) & $4,8-6$ & 5,5 \\
\hline TSH (mU/L) & $0,3-4$ & 3 \\
\hline FT4 (pmol/L) & $10-25$ & 17 \\
\hline Total Protein (g/dl) & $6,4-8,3$ & 7 \\
\hline Albumin (g/dl) & $3,5-5,2$ & 4,1 \\
\hline Total Bilirubin (mg/dl) & $0,3-1,2$ & 0,47 \\
\hline Direct Bilirubin (mg/dl) & $0-0,3$ & 0,14 \\
\hline Sodium (mMol/L) & $136-145$ & 144 \\
\hline Potassium (mMol/L) & $3,5-5,1$ & 4,2 \\
\hline CRP (mg/dl) & $0-5$ & 15,3 \\
\hline ESR (mm) & $0-20$ & 38 \\
\hline
\end{tabular}

\section{Commentary}

Autoimmune hepatitis is a chronic self-perpetuating inflammatory disease occurring in all ages and races [17]. Besides its diverse presentation and heterogeneity of the clinical features, AIH is characterized biochemically by elevated transaminases, histologically by interface hepatitis, and serologically by increased levels of immunoglobulin $\mathrm{G}$
(IgG) and autoantibodies, in the absence of viral markers [17]. Based on the autoantibody profile, two types of $\mathrm{AIH}$ are recognized: type 1 (AIH-1), positive for ANA and/or anti-smooth muscle antibody (SMA), and occurring at any age, and type 2 (AIH-2), positive for anti-liver-kidney microsomal antibody (anti-LKM) or for anti-liver cytosol type-1 antibody (anti-LC-1), with a peak incidence in children and adolescents [17-19]. AIH generally responds to immunosuppressive treatment, but if left untreated usually progresses to liver failure requiring transplantation [17].

Extra-hepatic disorders, mainly autoimmune conditions, are common in autoimmune hepatitis and occur in all stages of liver disease, including ulcerative colitis, Crohn's disease, vasculitis, arthritis, thyroiditis, diabetes mellitus, autoimmune hemolytic anemia, glomerulonephritis, fibrosing alveolitis, systemic lupus erythematosus, coeliac disease, sicca syndrome, vitiligo, or lymphoproliferative syndromes [20, 21].

On the other hand, PG is associated with a variety of mainly autoimmune diseases in 50-70\% of cases, with inflammatory bowel disease (IBD) topping the list at 10-15\% [6]. However, there is a dearth of data on the association of PG with AIH, as a search of the English-language literature unearthed only fifteen other cases [15].

Our patient had AIH-1, as the majority of reported cases when the type of $\mathrm{AIH}$ was identified, although AIH-2 may be underestimated [15]. Our patient's gender, the age at diagnosis of $\mathrm{AIH}$, and the interval to the subsequent onset of PG concur with those of the other reported cases, as does the development of PG during a quiescent stage of the AIH. Our patient remained in remission subsequently, even with the use of potentially hepatotoxic agents. Although a fulminant presentation of AIH has been proposed as a possible risk factor for the subsequent development of PG [15], our patient's hepatitis course was not severe, emphasizing our poor understanding of the pathogenesis of PG in relation to AIH. Finally, pathergy, although rare [15], is a hallmark of PG, and our patient's first appearance of an ulcer was

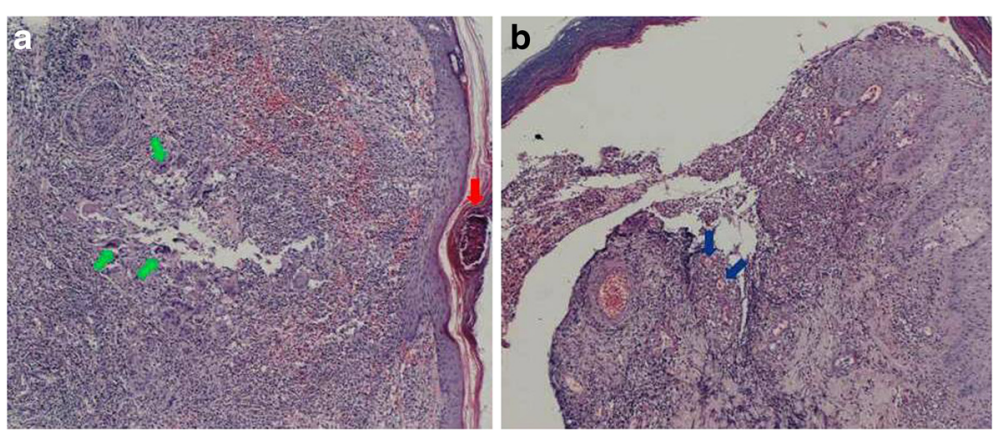

Fig. 2 Images of skin biopsy. a Abscess formation in the epidermis (red arrow) with accumulation of neutrophils and granuloma (green arrows) formation in the dermis. b The edge of an ulcer: deposits of eosinophilic material on the wall of the small blood vessels (blue arrow) 
at a previously "scratched" area, raising the distinct possibility of such event.

\section{Conclusion}

In summary, we report a patient with autoimmune hepatitis and pyoderma gangrenosum treated successfully with cortisone, cyclosporine, and methotrexate. Thorough testing excluded concurrent autoimmune diseases, confirming the association of pyoderma gangrenosum with autoimmune hepatitis, even when the latter is inactive. Clearly, the diagnosis of PG should raise the possibility of concomitant $\mathrm{AIH}$, since its diagnosis can be challenging and the results of failure to treat it devastating. On the other hand, AIH should sensitize us to the possible future development of PG. Overall, our case adds to the pool of knowledge about the presentation and management of PG in association with $\mathrm{AIH}$.

\section{Consent}

Written consent was obtained from the patient for publication of this Case report and any accompanying images. A copy of the written consent is available for review by the Editor of this journal.

\begin{abstract}
Abbreviations
PG: Pyoderma gangrenosum; IBD: Inflammatory bowel disease: AlH: Autoimmune hepatitis; ANA: Anti-nuclear antibodies; ASMA: Antismooth muscle antibodies; MRI: Magnetic resonance imaging; MRCP: Magnetic resonance cholangiopancreatography; Anti-LKM: Anti-liver kidney microsomal antibodies; Anti-LC-1: Anti-liver cytosol type 1 antibodies; IgG: Immunoglobulin G.

\section{Competing interests}

The authors declare that they have no competing interests.

Authors' contribution

TA took care of the patient, collected all data and clinical images, and drafted the manuscript. GH was the hepatologist in charge of the patient and contributed in the drafting of the manuscript. PS provided surgical expertise about the patient's ulcers and took the biopsies from the lesions. KA was the pathologist that examined the biopsy and provided the biopsy pictures. All authors read and approved the final manuscript.
\end{abstract}

\section{Acknowledgements}

The authors thank our patient for her participation and her consent to publish these data. We also wish to thank Professor Athanasios Tzioufas, Director of Department of Pathophysiology of Laiko General Hospital, for his help in the diagnosis and treatment of the patient.

\footnotetext{
Author details

'Pathophysiology Department, Laiko General Hospital, National and Kapodistrian University of Athens, Medical School, Athens, Greece. ${ }^{2}$ Second Department of Propedeutic Surgery, Laiko General Hospital, National and Kapodistrian University of Athens, Medical School, Athens, Greece. ${ }^{3}$ Department of Dermatopathology, Medical School, National and Kapodistrian University of Athens, Athens, Greece.
}

Received: 20 July 2015 Accepted: 14 October 2015

Published online: 26 October 2015

\section{References}

1. Su WP, Davis MD, Weenig RH, et al. Pyoderma gangrenosum: clinicopathologic correlation and proposed diagnostic criteria [review]. Int J Dermatol. 2004;43:790-800. doi:10.1111/j.1365-4632.2004.02128.x. PMID: 15533059.
2. Powell FC, Su WP, Perry HO. Pyodermagangrenosum classification and management. J Am Acad Dermatol. 1996;34:395-409. doi:10.1016/501909622(96)90428-4. PMID: 8609250.

3. Wollina U. Pyodermagangrenosum — a review. Orphanet J Rare Dis. 2007;2:19. doi:10.1186/1750-1172-2-19.

4. Su WP, Schroeter AL, Perry HO, Powell FC. Histopathologic and immunopathologic study of pyodermagangrenosum. J CutanPathol. 1986;13:323-30. PMID: 3537032.

5. Callen JP. Pyodermagangrenosum. Lancet. 1998;351:581-5. doi:10.1016/ S0140-6736(97)10187-8. PMID: 9492798.

6. Bernstein CN, Blanchard JF, Rawsthorne P, et al. The prevalence of extraintestinal diseases in inflammatory bowel disease: a population based study. Am J Gastroenterol. 2001;96:1116-22. doi:10.1111/j.1572-0241.2001.03756.x. PMID: 11316157.

7. Stolman LP, Rosenthal D, Yaworsky R, et al. Pyoderma gangrenosum and rheumatoid arthritis. Arch Dermatol. 1975;111:1020-3. doi:10.1001/ archderm.1975.01630200080009. PMID: 786171.

8. Powell FC, Schroeter AL, Su WP, et al. Pyoderma gangrenosum: a review of 86 patients. Q J Med. 1985:55:173-86. PMID: 3889978.

9. Török L, Kirschner A, Gurzo M, et al. Bullous pyoderma gangrenosum as a manifestation of leukemia cutis. Eur J Dermatol. 2000;10:463-5. PMID: 10980469.

10. Wayte JA, Rogers S, Powell FC. Pyoderma gangrenosum, erythema elevatum diutinum and IgA monoclonal gammopathy. Australas J Dermatol. 1995;36:21-3. doi:10.1111/j.1440- 0960.1995.tb00919.x. PMID: 7763217.

11. Keane FM, MacFarlane CS, Munn SE, Higgins EM. Pyoderma gangrenosum and hepatitis C virus infection. Br J Dermatol. 1998;139:924-5. doi:10.1046/ j.1365.1998.02531.x. PMID: 9892972.

12. Goldenberg G, Jorizzo JL. Use of etanercept in treatment of pyoderma gangrenosum in a patient with autoimmune hepatitis. J Dermatol Treat. 2005;16(5-6):347-9. doi:10.1080/09546630500424722. PMID: 16428159.

13. Koskinas J, Raptis I, Manika Z, et al. Overlapping syndrome of autoimmune hepatitis and primary sclerosing cholangitis associated with pyoderma gangrenosum and ulcerative colitis. Eur J Gastroenterol Hepatol. 1999;11:1421-4. doi:10.1097/00042737-199912000-00014. PMID: 10654805.

14. Halac U, Dhaybi RA, Powell J, Soglio DB-D, Alvarez F. Unusual association between autoimmune hepatitis and severe pyoderma gangrenosum. JPGN. 2010;50:219-21. doi:10.1097/MPG.0b013e31819a62f5. PMID: 19590450.

15. Wat $\mathrm{H}$, Haber RM. Trends in the association between Pyoderma Gangrenosum and Autoimmune Hepatitis: Case report and Review of the Literature. J Cutan Med Surg. 2014;18(5):345-52. doi:10.2310/ 7750.2013.13177. PMID: 25186997.

16. Miller J, Yentzer BA, Clark A, Jorizzo JL, Feldman SR. Pyoderma gangrenosum: A review and update on new therapies. J Am Acad Dermatol. 2010;62:646-54. doi:10.1016/j.jaad.2009.05.030. PMID: 20227580

17. Manns MP, Lohse AW, Vergani D. Autoimmune hepatitis - Update 2015. J Hepatol. 2015;62(1 Suppl):S100-11. doi:10.1016/j.jhep.2015.03.005. PMID: 25920079

18. Liberal R, Grant CR, Mieli-Vergani G, Vergani D. Autoimmune hepatitis: a comprehensive review. J Autoimmun. 2013;41:126-39. doi:10.1016/ j.jaut.2012.11.002. PMID: 23218932.

19. Vierling JM. Autoimmune hepatitis and overlap syndromes: Diagnosis and Management. Clin Gastroenterol Hepatol 2015; PMID: 26284592 DOI: 10.1016/j.cgh.2015.08.012

20. Muratori P, Fabbri A, Lalanne C, Lenzi M, Muratori L. Autoimmune liver disease and concomitant extrahepatic autoimmune disease. Eur J Gastroenterol Hepatol. 2015;27(10):1175-9. doi:10.1097/ MEG.0000000000000424. PMID: 26148248.

21. Teufel A, Weinmann A, Kahaly GJ, Centner C, Piendl A, Worns M, et al. Concurrent autoimmune diseases in patients with autoimmune hepatitis. J Clin Gastroenterol. 2010;44:208-13. doi:10.1097/MCG.0b013e3181c74e0d. PMID: 20087196 